\title{
Upshot of Music-Blended Teaching on Anxiety, Interest and Performance of Students with Mathematics Learning Disability
}

\author{
B. William Dharma Raja \\ Department of Education, Manonmaniam Sundaranar University, Tirunelveli, India
}

Email address:

widh07@yahoo.com

To cite this article:

B. William Dharma Raja. Upshot of Music-Blended Teaching on Anxiety, Interest and Performance of Students with Mathematics Learning Disability. Science Journal of Education. Vol. 5, No. 4, 2017, pp. 164-173. doi: 10.11648/j.sjedu.20170504.17

Received: March 7, 2017; Accepted: April 6, 2017; Published: July 6, 2017

\begin{abstract}
Music is concerned with the physical and emotional wellbeing of any human being. Listening to pleasant music in the background while doing an arduous task can make it seem so much easier, or in some cases, music may not increase positive attitude, but will ease the strain of an activity. This study assessed whether music blended teaching helps the learners who face difficulties while learning mathematics. The sample consisted of 42 students identified with mathematics learning disability. The experimental group was taught mathematics with violin instrumental music played in the background of the Mathematics classroom. The control group was taught through conventional teaching. The treatment lasted for 44 days, 45 minutes per day. For collection of relevant data, the investigator used two standardised tools namely, Coloured Progressive Matrices (CPM) and PrWi's Mathematical Ability Test (PMAT). The self-made tools were: Mathematics Achievement Test (MAT), Scale on Interest in Mathematics (SIM) and Mathematics test Anxiety Scale (MAS). The pre and post-test analyses disclosed that the experimental group students exhibit lesser mathematics test anxiety in the post-test than the pre-test. In the gain scores it was found that both the control and experimental groups did not show any difference in the achievement, interest and anxiety scores in mathematics. In delayed post-test, the experimental group showed better performance in the learning objective - knowledge than the control group. Also the experimental group students with high level intelligence exhibited better scores in the learning objective - knowledge than the counter parts in control group. No significant difference was found in the delayed post-test in interest and anxiety of both the control and experimental groups. It is also true with regard to the level of intelligence.
\end{abstract}

Keywords: Music, Mathematics Learning Disability, Interest in Mathematics, Mathematics Anxiety

\section{Introduction}

Music, a magical medium in every aspect of our life [7], is scientifically proven that music has influence on cognitive skills, learning, working memory, way of thinking, and personal and social development [35] (Urbantimes, n. d.). Background music is used commonly in restaurants, waiting rooms, elevators and other public places to reduce stress and anxiety or to facilitate desired behaviours. Background music can be defined as any music played while the listener's attention is focused primarily on a task or activity in which the listener is involved. Such a task or activity could be studying or other academic preparation. Students of all ages have often claimed that they can learn more effectively while listening to music [5]. Music can create a sense of playfulness and joy in the classroom. It can bring about a feeling of freedom as students search for and create unique patterns and rhythms. This helps create an atmosphere that encourages emotional well-being within a positive learning environment [1]. Every teacher likes to bring joyful learning in their classrooms.

\section{Importance of the Study}

The right music at the right time alters or affects the brain waves. Certain musical sound waves, especially the western classical violin instrumental music helps to hold the attention and intake more information in the highly focused state. The 
violin music can help to shift the wave of students' brain from alpha state to beta state, which is a perfect learning state for taking in information through auditory channels occur. This music further evokes emotions and stimulates involvement in learning. For great understanding, teachers can use music intentionally when memory activities happen.

The physical vibration of music and its organized patterns of sound, rhythm, and subtle vibrations interact with the nervous system of the body and result in the alteration of the human behaviour in many ways, nearly every region of the brain and neural subsystem. Listening to music can influence the frequency of brain waves and so the state of mind. It not only affects the mind but also changes the state of the body.

Music can link the mental and physical self which directly affects the working memory. The cerebral cortex self organizes when an individual engages with musical activities. Music synchronizes the right and left hemispheres of the brain. The left hemisphere analyses the structure of music, while the right hemisphere focuses on the melody. When listening to music, the hemispheres of the brain work together; emotions are stimulated; attention is focused and motivation is heightened. Music acts as an anchor for capturing attention and stimulating interest inside the classroom. Research suggests that music can actually hardwire the brain, which build links between the two hemispheres of the brain and be utilized for a variety of cognitive activities. Researchers believe that children who have more exposure to music and music training benefit from enhanced brain activity. This ability to perform certain academic tasks like preparing for examinations and enhance recalling shapes or pictures has been increased. Music induces a receptive mood that generally enhances cognitive process. It can serve as a mnemonic memory aid to encode information in the mind and support initial learning to recall and transference of working memory. Music, which is one of the factors affect memory, is a stimulator for many parts of the brain. Music stimulates the areas of the brain that involves in emotion, association and long-term memory processes.

An individual has a specific 'learning biography' which is reflected in the way the brain processes information. As individuals engage with different musical activities over a long period of time, permanent changes occur in the brain. These reflect what has been learned and how it has been learned. They will also influence the extent to what extent the developed skills are able to transfer to other activities. It is also beneficial to the development of social skills and can contribute to health and well-being throughout the lifespan and therefore can contribute to community cohesion providing benefits to society as a whole.

Music-Mathematics blended classroom is to establish good distraction to block the bad one, which will unambiguously result in positive findings. It helps to establish and increase students' ability to concentrate and focus. Furthermore, using background music inside the classroom may help learners and teachers to create an impingement achievement, interest and anxiety. It will enable brainwork such as analytical, creative and aspects of motivation. Music has the neutral firepower to jazz up the thought process. Music blended math classroom of primary students can induce concentration, filter out any distractions and structure the thoughts of the dissimilar students. This technique is an addition to the acoustics of the classroom which will help the dissimilar mathematics learners to encode, decode, process and improve the recall process with an ease. This study will be relevance for teachers who deal with primary students with mathematics learning disability (MLD) in their classroom. Teachers need to remove negative threats and factors in the learning environment. They can try to introduce music in the Mathematics classroom as a positive stimulant. It can enhance achievement, foster interest and take away anxiety from the primary students who found with MLD. It can make the Mathematics classrooms a joyful learning place.

In the classroom music helps the learners to get the right mental state for learning; it helps the teacher to get the right mental state for teaching; it acts as an anchor and a direct link to feelings and emotions; it shows to be effective in accelerated learning; it helps with motivation, as a way of celebrating good work or behaviour; it improves memory; and it acts as a cause to release dopamine-the learning neurochemical (Ian Gilbert, 2009 in [20] Jackson, 2009).

\section{Literature Review}

The related studies carried out for more than a decade (2001 - 2015), which are synthesized below.

The reviewed studies imply that five to three years participation in music ([36] Whitehead, 2002; [8]; [29] Olson, 2004; [25] McLelland, 2005), and listening to classical music [26] (Merrell, 2004; Jones \& Estell, 2006; Jones \& Estell, 2007) had significant impact on performance in mathematics. Contrary to these studies, there are studies which found that participation in music instruction (Kluball, 2001) and listening to classical music ([13]Furnhan \& Strbac, 2002; Goldenberg et al., 2013) had no positive effect on performance in mathematics.

Music had its positive impact on other subjects/areas such as reading (Furnhan,2002; Cardarelli, 2004; [29] Olson, 2004; [25] McLelland, 2005; [12] Eaton, 2007), spelling [3], and language skills [22]. There are contradictory studies also which revealed that music had no effect on reading [11] (Doyle \& Furnham, 2012), writing [23](Kluball, 2001), language skills [10](Costa, 2004) and English, Science and Social Science [23] (Kluball, 2001) as well.

Exposure to music enhanced recall and memory [6] and cognitive processing [32] (Roden et al., 2013). It is also revealed that music instruction enhances IQ [28] (Nering, 2002; [33] Schellenberg, 2004) and affects the behavioural effects of children enrolled in special education [24] (Lang, 2001). These findings were not supported by the study of Furnhan (2002) which reveals that no significant interaction of music was found on recall and study by Charmorro et al.(2009) reveals that no significant influence of music on cognitive task performance. 
Background music reduces mathematic anxiety [16] test anxiety [30] (Rastogi, 2014) and depression (Hendricks, 2001), and increases well-being [18]. In contradiction, Goldenberg et al. (2013) found that listening to music did not decrease test anxiety.

Of the reviewed studies, only two studies ([27] Montello, 1998; [32] Savan, 1998) were conducted to know the impact of music on students with learning, emotional and behavioural disorders. But no studies were conducted on the impact of music on students with MLD. Apart from studies done with normal children, two studies were on mathematics interest [2] and a few studies (Hendricks, 2001; Haynes, 2003; Hirokawa \& Ohira, 2003; Goldenberg et al., 2013; Rustogi, 2014) on the anxiety of the students. It was hard to find studies conducted exclusively on music and students with MLD. This piece of research was attempted to study the achievement, interest and test anxiety of the primary students in connection with mathematics who found with Mathematics learning disability.

\section{Objectives of the Study}

The objectives of the study are:

a) To study the effectiveness of music-fused-teaching on the gain in achievement, interest and anxiety of primary students with mathematics learning disability.

b) To find out the significant difference, if any, between the control and experimental groups in the achievement, interest and anxiety of primary students with mathematics learning disability in the delayed post test scores.

c) To identify the influence, if any, of intelligence on the achievement, interest and anxiety of primary students with mathematics learning disability.

Hypotheses Framed

In the light of the objectives, the following hypotheses were formulated.

a) There is no significant difference in the achievement in mathematics, mathematics interest and mathematics anxiety between the control and experimental groups of primary students with mathematics learning disability before treatment.

b) There is significant difference in the achievement in mathematics, interest in mathematics and mathematics anxiety between the control and experimental groups of primary students with mathematics learning disability after treatment.

c) There is significant difference in delayed posttest scores of achievement in mathematics, interest in mathematics and mathematics anxiety between the control and experimental groups of primary students with mathematics learning disability.

Methodology

a) Sample

Forty two students of Standard V, out of 455, from a Matriculation Higher Secondary School in Kanyakumari District, India, scored less than 33\% in PrWi's Mathematics
Ability Test (PMAT) were chosen as sample for the study.

b) Design of the study

Pre-test and post-test equivalent Group Design [4] was used in this study. The students with MLD were identified and selected for the control and experimental groups. The students of the two groups were equivalent with regard to intelligence and mathematical ability.

The study was conducted with six stages.

Stage 1 - Identifying primary students with MLD. PMATwas administered to 455 students of a Higher Secondary School located in Kanyakumari district, Indiato identify the mathematical ability of them in the areas such as: concept of time, fractions, place value and decimals, identifying correct sign, correct shape, smallest or largest number and correct symbol, spatial ability, computation ability, ability to use Mathematical language, sequential ability, counting ability, perceptual ability and reasoning ability. Students who scored less than $33 \%$ were considered to have MLD and they were 42.

Stage 2 - Grouping the students with MLD into equivalent group. Raven's Coloured Progressive Matrices (CPM) was administered to the identified students with MLD to find out their intelligence level. Based on the intelligence, 42 students with MLD were divided into control and experimental groups, 21 students each.

Stage 3 - Conducting pre-test for the sample groups. The self-made tools namely, Mathematics Achievement Test (MAT), Scale on Interest in Mathematics (SIM) and Mathematics test Anxiety Scale (MAS) were administered for both the control and experimental groups before the treatment.

Stage 4 - Treatment to the sample groups. The experimental group students were taught mathematics with violin instrumental music played in the background of the Standard V - Mathematics classroom. The description of the violin pieces exercised during the treatment to the experimental group is appended. The control group students were taught through conventional teaching. The treatment lasted for 44 days/45 minutes/day.

Stage 5 - Conducting post-test for the sample groups. Immediately after the treatment period, the tools - MAT, SIM, MAS were administered again for both the control and experimental groups.

Stage 6 - Conducting delayed post-test for the sample groups. The above three tools were again administered to the sample groups after a period of two weeks from the post-test to measure the retention capacity.

c) Description of the Self-made Tools

For MAT, items having difficulty value between 40 and 80 and discriminative index greater than 0.2 (Stanley, 1978) [34] were selected and the remaining items were left out. The reliability of MAT was calculated by the Spearman-Brown Prophecy formula [14] (Garrett, 1961) and the reliability coefficient was found to be 0.73 which indicates that MAT is highly reliable [4] (Best \& Kahn, 2012).

For establishing the content validity of SIM, it was given to three senior teacher educators for affirming its content 
validity. In order to find the item validity of SIM, the item total correlation was found. The r-value of items in the preliminary draft of SIM equal to 0.26 or above were selected. The reliability coefficient of SIM by split-half method was found to be 0.62 . From the self-correlation of the half-test, the reliability coefficient of the whole test $r^{\prime}$ found by the Spearman-Brown Prophecy formula was 0.76 which indicates that the SIM is highly reliable [4] (Best \& Kahn, 2012).

To ensure the suitability of the framed items in MAS, it was submitted to three experts in Mathematics education and early childhood education. The items in the MAS were pruned for avoiding ambiguity. Thus MAS had 22 items. Then item total correlation was found and all the items were with ' $r$ ' value greater than 0.4. Co-efficient of reliability, calculated using the Spearman-Brown Prophecy formula, was found to be 0.84 which indicated that MAS is highly reliable.

d) Statistical techniques employed

$t$ test for independent, small groups and $t$ test for dependent groups were employed for the study.

\section{Results}

The collected data were analysed using the parametric tests, namely t tests for independent and dependent means, and the results are given in the tables 1-9.

Table 1. Difference in Pretest scores in the attributes of sample students with MLD.

\begin{tabular}{|c|c|c|c|c|c|c|}
\hline Dependent Variables & & Group & Mean & SD & t value & P value \\
\hline & Knowledge & $\begin{array}{l}\text { Control } \\
\text { Exptl. }\end{array}$ & $\begin{array}{l}52.381 \\
5.020\end{array}$ & $\begin{array}{l}22.284 \\
22.844\end{array}$ & 0.195 & $0.846^{\mathrm{NS}}$ \\
\hline Achievement in Mathematics & Understanding & $\begin{array}{l}\text { Control } \\
\text { Exptl. }\end{array}$ & $\begin{array}{l}26.667 \\
28.095\end{array}$ & $\begin{array}{l}12.780 \\
13.274\end{array}$ & 0.355 & $0.724^{\mathrm{NS}}$ \\
\hline & Application & $\begin{array}{l}\text { Control } \\
\text { Exptl. }\end{array}$ & $\begin{array}{l}31.933 \\
35.294\end{array}$ & $\begin{array}{l}10.452 \\
11.617\end{array}$ & 0.986 & $0.330^{\mathrm{NS}}$ \\
\hline Interest in Mathematics & & $\begin{array}{l}\text { Control } \\
\text { Exptl. }\end{array}$ & $\begin{array}{l}72.714 \\
74.111\end{array}$ & $\begin{array}{l}8.914 \\
9.542\end{array}$ & 0.491 & $0.626^{\mathrm{NS}}$ \\
\hline Mathematics test Anxiety & & $\begin{array}{l}\text { Control } \\
\text { Exptl. }\end{array}$ & $\begin{array}{l}50.721 \\
52.525\end{array}$ & $\begin{array}{l}10.953 \\
10.325\end{array}$ & 0.549 & $0.586^{\mathrm{NS}}$ \\
\hline
\end{tabular}

NS - Not Significant

Table 2. Difference in Posttest scores in the attributes of sample students with MLD.

\begin{tabular}{|c|c|c|c|c|c|c|}
\hline Dependent Variables & & Group & Mean & SD & t value & $P$ value \\
\hline & Knowledge & $\begin{array}{l}\text { Control } \\
\text { Exptl. }\end{array}$ & $\begin{array}{l}72.789 \\
72.109\end{array}$ & $\begin{array}{c}22.544 \\
9.557\end{array}$ & 0.127 & $0.899^{\mathrm{NS}}$ \\
\hline Achievement in Mathematics & Understanding & $\begin{array}{l}\text { Control } \\
\text { Exptl. }\end{array}$ & $\begin{array}{l}44.286 \\
43.809\end{array}$ & $\begin{array}{l}20.142 \\
14.655\end{array}$ & 0.088 & $0.931^{\mathrm{NS}}$ \\
\hline & Application & $\begin{array}{l}\text { Control } \\
\text { Exptl. }\end{array}$ & $\begin{array}{l}43.417 \\
41.176\end{array}$ & $\begin{array}{l}15.166 \\
12.616\end{array}$ & 0.521 & $0.606^{\mathrm{NS}}$ \\
\hline Interest in Mathematics & & $\begin{array}{l}\text { Control } \\
\text { Exptl. }\end{array}$ & $\begin{array}{l}73.960 \\
75.246\end{array}$ & $\begin{array}{l}11.813 \\
12.271\end{array}$ & 0.346 & $0.731^{\mathrm{NS}}$ \\
\hline Mathematics test Anxiety & & $\begin{array}{l}\text { Control } \\
\text { Exptl. }\end{array}$ & $\begin{array}{l}50.865 \\
45.671\end{array}$ & $\begin{array}{l}16.991 \\
14.817 \\
\end{array}$ & 1.056 & $0.297^{\mathrm{NS}}$ \\
\hline
\end{tabular}

Table 3. Difference between pre-test and post-test scores if the attributes of students in the control group.

\begin{tabular}{|c|c|c|c|c|c|c|c|}
\hline Dependent Variables & & Test & Mean & SD & R value & t value & P value \\
\hline \multirow{3}{*}{ Achievement in Mathematics } & Knowledge & $\begin{array}{l}\text { Pre } \\
\text { Post }\end{array}$ & $\begin{array}{l}52.380 \\
72.789\end{array}$ & $\begin{array}{l}22.284 \\
22.544\end{array}$ & 0.379 & 3.744 & $0.001^{* *}$ \\
\hline & Understanding & $\begin{array}{l}\text { Pre } \\
\text { Post }\end{array}$ & $\begin{array}{l}26.667 \\
44.286\end{array}$ & $\begin{array}{l}12.780 \\
20.142\end{array}$ & 0.583 & 4.922 & $0.000^{* *}$ \\
\hline & Application & $\begin{array}{l}\text { Pre } \\
\text { Post }\end{array}$ & $\begin{array}{l}31.923 \\
43.417\end{array}$ & $\begin{array}{l}10.452 \\
15.166\end{array}$ & 0.246 & 3.257 & $0.004^{*}$ \\
\hline Interest in Mathematics & & $\begin{array}{l}\text { Pre } \\
\text { Post }\end{array}$ & $\begin{array}{l}70.748 \\
71.315\end{array}$ & $\begin{array}{l}10.133 \\
11.939\end{array}$ & 0.004 & 0.166 & $0.870^{\mathrm{NS}}$ \\
\hline Mathematics test Anxiety & & $\begin{array}{l}\text { Pre } \\
\text { Post }\end{array}$ & $\begin{array}{l}50.723 \\
50.866\end{array}$ & $\begin{array}{l}10.953 \\
16.991\end{array}$ & 0.350 & 0.040 & $0.969^{\mathrm{NS}}$ \\
\hline
\end{tabular}

** Significant at $1 \%$ level * Significant at $5 \%$ level NS - Not Significant

Table 4. Difference between pre-test and post-test scores in the attributes of students in the control group with regard to level of intelligence.

\begin{tabular}{|c|c|c|c|c|c|c|c|c|}
\hline Level of Intelligence & Dependent Variables & & Test & Mean & SD & r value & t value & $P$ value \\
\hline \multirow{4}{*}{ Low } & \multirow{4}{*}{$\begin{array}{l}\text { Achievement in } \\
\text { Mathematics }\end{array}$} & \multirow{2}{*}{ Knowledge } & Pre & 47.619 & 29.738 & \multirow{2}{*}{0.350} & \multirow{2}{*}{1.964} & \multirow{2}{*}{$0.188^{\mathrm{NS}}$} \\
\hline & & & Post & 90.476 & 35.952 & & & \\
\hline & & \multirow{2}{*}{ Understanding } & Pre & 20.000 & 10.000 & \multirow{2}{*}{1.000} & \multirow{2}{*}{1.732} & \multirow{2}{*}{$0.225^{\mathrm{NS}}$} \\
\hline & & & Post & 40.000 & 30.000 & & & \\
\hline
\end{tabular}




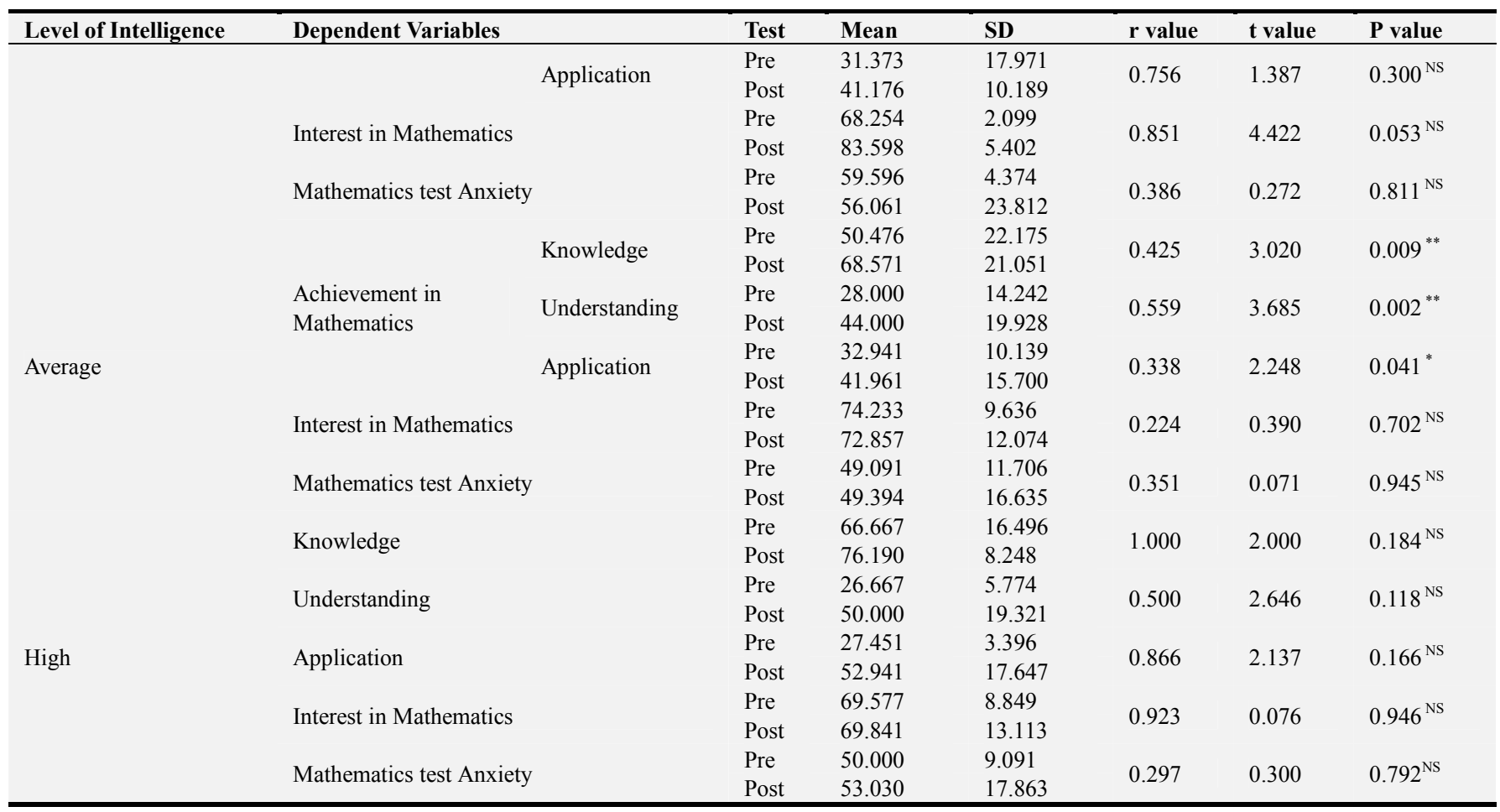

Table 5. Difference between pre-test and post-test scores in the attributes of students in the experimental group.

\begin{tabular}{|c|c|c|c|c|c|c|c|}
\hline \multicolumn{2}{|c|}{ Dependent Variables } & Test & Mean & SD & $R$ value & t value & P value \\
\hline \multirow{3}{*}{$\begin{array}{l}\text { Achievement in } \\
\text { Mathematics }\end{array}$} & Knowledge & $\begin{array}{l}\text { Pre } \\
\text { Post }\end{array}$ & $\begin{array}{l}51.020 \\
72.109\end{array}$ & $\begin{array}{l}22.844 \\
9.558\end{array}$ & 0.300 & 4.402 & $0.000^{* *}$ \\
\hline & Understanding & $\begin{array}{l}\text { Pre } \\
\text { Post }\end{array}$ & $\begin{array}{l}28.095 \\
43.809\end{array}$ & $\begin{array}{l}13.274 \\
14.655\end{array}$ & 0.193 & 4.053 & $0.001^{* *}$ \\
\hline & Application & $\begin{array}{l}\text { Pre } \\
\text { Post }\end{array}$ & $\begin{array}{l}35.294 \\
41.176\end{array}$ & $\begin{array}{l}11.617 \\
12.616\end{array}$ & 0.744 & 3.090 & $0.006^{* *}$ \\
\hline \multicolumn{2}{|c|}{ Interest in Mathematics } & $\begin{array}{l}\text { Pre } \\
\text { Post }\end{array}$ & $\begin{array}{l}74.112 \\
75.246\end{array}$ & $\begin{array}{l}9.542 \\
12.271\end{array}$ & 0.450 & 0.445 & $0.661^{\mathrm{NS}}$ \\
\hline \multicolumn{2}{|c|}{ Mathematics test Anxiety } & $\begin{array}{l}\text { Pre } \\
\text { Post }\end{array}$ & $\begin{array}{l}52.525 \\
45.671\end{array}$ & $\begin{array}{l}10.325 \\
14.817\end{array}$ & 0.650 & 2.784 & $0.011 *$ \\
\hline
\end{tabular}

Table 6. Difference between pre-test and post-test scores in the attributes of students in the experimental group with regard to level of intelligence.

\begin{tabular}{|c|c|c|c|c|c|c|c|c|}
\hline Level of Intelligence & Dependent Vari & les & Test & Mean & SD & r value & t value & P value \\
\hline \multirow{8}{*}{ Low } & \multirow{5}{*}{$\begin{array}{l}\text { Achievement in } \\
\text { Mathematics }\end{array}$} & Knowledge & $\begin{array}{l}\text { Pre } \\
\text { Post }\end{array}$ & $\begin{array}{l}46.429 \\
67.857\end{array}$ & $\begin{array}{l}29.451 \\
7.143\end{array}$ & -0.566 & 1.260 & $0.297^{\mathrm{NS}}$ \\
\hline & & \multirow{2}{*}{ Understanding } & Pre & 27.500 & 9.574 & \multirow{2}{*}{0.135} & \multirow{2}{*}{1.000} & \multirow{2}{*}{$0.391^{\mathrm{NS}}$} \\
\hline & & & Post & 35.000 & 12.909 & & & \\
\hline & & \multirow[t]{2}{*}{ Application } & Pre & 36.765 & 10.046 & \multirow[t]{2}{*}{0.956} & \multirow[t]{2}{*}{1.567} & \multirow[t]{2}{*}{$0.215^{\mathrm{NS}}$} \\
\hline & & & Post & 41.576 & 14.409 & & & \\
\hline & \multirow{2}{*}{\multicolumn{2}{|c|}{ Interest in Mathematics }} & Pre & 73.809 & 8.861 & \multirow{2}{*}{0.059} & \multirow{2}{*}{0.765} & \multirow{2}{*}{$0.500^{\mathrm{NS}}$} \\
\hline & & & Post & 78.769 & 10.005 & & & \\
\hline & \multicolumn{2}{|c|}{ Mathematics test Anxiety } & $\begin{array}{l}\text { Pre } \\
\text { Post }\end{array}$ & $\begin{array}{l}48.106 \\
43.561\end{array}$ & $\begin{array}{l}11.564 \\
20.455\end{array}$ & 0.808 & 0.697 & $0.536^{\mathrm{NS}}$ \\
\hline \multirow{10}{*}{ Average } & \multirow{6}{*}{$\begin{array}{l}\text { Achievement in } \\
\text { Mathematics }\end{array}$} & \multirow{2}{*}{ Knowledge } & Pre & 47.959 & 21.415 & \multirow{2}{*}{0.397} & \multirow{2}{*}{4.639} & \multirow{2}{*}{$0.000^{* *}$} \\
\hline & & & Post & 72.449 & 10.429 & & & \\
\hline & & \multirow{2}{*}{ Understanding } & Pre & 25.000 & 10.919 & \multirow{2}{*}{0.000} & \multirow{2}{*}{4.169} & \multirow{2}{*}{$0.001^{* *}$} \\
\hline & & & Post & 45.714 & 15.045 & & & \\
\hline & & \multirow{2}{*}{ Application } & Pre & 36.554 & 11.805 & \multirow{2}{*}{0.637} & \multirow{2}{*}{2.645} & \multirow{2}{*}{$0.020^{*}$} \\
\hline & & & Post & 43.697 & 11.925 & & & \\
\hline & \multirow{2}{*}{\multicolumn{2}{|c|}{ Interest in Mathematics }} & Pre & 73.583 & 10.531 & 0471 & 0070 & $045 \mathrm{NS}$ \\
\hline & & & Post & 73.356 & 12.773 & $0.4 / 1$ & $0.0 / 0$ & 0.945 \\
\hline & Mathematics test & nxiety & Pre & 53.896 & 10.237 & 0.615 & 2879 & $0013 *$ \\
\hline & Mathematics test & nxiety & Post & 45.238 & 14.137 & 0.615 & $2.8 / 9$ & $0.013^{*}$ \\
\hline & Knowledore & & Pre & 71.428 & 14.286 & 0866 & $10 \Omega 0$ & $0423^{\mathrm{NS}}$ \\
\hline High & Knowledge & & Post & 76.190 & 8.248 & 0.866 & 1.000 & 0.423 \\
\hline & Understanding & & Pre & 43.333 & 20.817 & 0.996 & 1.000 & $0.423^{\mathrm{NS}}$ \\
\hline
\end{tabular}




\begin{tabular}{|c|c|c|c|c|c|c|c|}
\hline Level of Intelligence & Dependent Variables & Test & Mean & SD & r value & t value & $P$ value \\
\hline & \multirow{3}{*}{ Application } & Post & 46.667 & 15.275 & \multirow{3}{*}{1.000} & \multirow{3}{*}{1.000} & \multirow{3}{*}{$0.423^{\mathrm{NS}}$} \\
\hline & & Pre & 27.451 & 13.585 & & & \\
\hline & & Post & 29.412 & 10.188 & & & \\
\hline & \multirow{2}{*}{ Interest in Mathematics } & Pre & 76.984 & 7.571 & \multirow{2}{*}{0.755} & \multirow{2}{*}{0.397} & \multirow{2}{*}{$0.729^{\mathrm{N}}$} \\
\hline & & Post & 79.365 & 14.827 & & & \\
\hline & \multirow{2}{*}{ Mathematics test Anxiety } & Pre & 52.020 & 11.473 & \multirow{2}{*}{0.687} & \multirow{2}{*}{0.240} & \multirow{2}{*}{$0.833^{\mathrm{NS}}$} \\
\hline & & Post & 50.505 & 14.948 & & & \\
\hline
\end{tabular}

** - Significant at 1\% level * - Significant at 5\% level NS - Not Significant

Table 7. Difference in the gain scores in the attributes of students in the sample groups.

\begin{tabular}{|c|c|c|c|c|c|c|}
\hline Dependent Variables & & Group & Mean & SD & t value & $P$ value \\
\hline \multirow{6}{*}{ Achievement in Mathematics } & \multirow{2}{*}{ Knowledge } & Control & 20.408 & 24.978 & \multirow{2}{*}{0.289} & \multirow{2}{*}{$0.774^{\mathrm{NS}}$} \\
\hline & & Exptl. & 22.449 & 20.489 & & \\
\hline & \multirow{2}{*}{ Understanding } & Control & 17.619 & 16.403 & \multirow{2}{*}{0.361} & \multirow{2}{*}{$0.720^{\mathrm{NS}}$} \\
\hline & & Exptl. & 15.714 & 17.768 & & \\
\hline & \multirow{2}{*}{ Application } & Control & 11.485 & 16.161 & \multirow{2}{*}{1.398} & \multirow{2}{*}{$0.170^{\mathrm{NS}}$} \\
\hline & & Exptl. & 5.882 & 8.725 & & \\
\hline \multirow{2}{*}{ Interest in Mathematics } & & Control & 1.247 & 13.148 & \multirow{2}{*}{0.030} & \multirow{2}{*}{$0.977^{\mathrm{NS}}$} \\
\hline & & Exptl. & 1.133 & 11.668 & & \\
\hline \multirow{2}{*}{ Mathematics test Anxiety } & & Control & -0.144 & 16.679 & \multirow{2}{*}{0.515} & \multirow{2}{*}{$0.610^{\mathrm{NS}}$} \\
\hline & & Exptl. & 2.237 & 13.092 & & \\
\hline
\end{tabular}

NS - Not Significant

Table 8. Difference in the delayed post-test scores in the attributes of students in the sample groups.

\begin{tabular}{|c|c|c|c|c|c|c|}
\hline Dependent Variables & & Group & Mean & SD & t value & $P$ value \\
\hline \multirow{6}{*}{ Achievement in Mathematics } & \multirow{2}{*}{ Knowledge } & Control & 70.068 & 12.709 & \multirow{2}{*}{3.451} & \multirow{2}{*}{$0.001^{* *}$} \\
\hline & & Exptl. & 80.952 & 6.900 & & \\
\hline & \multirow{2}{*}{ Understanding } & Control & 40.476 & 16.575 & \multirow{2}{*}{0.272} & \multirow{2}{*}{$0.787^{\mathrm{NS}}$} \\
\hline & & Exptl. & 41.905 & 17.498 & & \\
\hline & \multirow{2}{*}{ Application } & Control & 42.017 & 17.179 & \multirow{2}{*}{0.168} & \multirow{2}{*}{$0.868^{\mathrm{NS}}$} \\
\hline & & Exptl. & 42.857 & 15.242 & & \\
\hline \multirow{2}{*}{\multicolumn{2}{|c|}{ Interest in Mathematics }} & Control & 76.455 & 14.082 & \multirow{2}{*}{0.521} & \multirow{2}{*}{$0.605^{\mathrm{NS}}$} \\
\hline & & Exptl. & 74.225 & 13.651 & & \\
\hline \multirow{2}{*}{ Mathematics test Anxiety } & & Control & 50.144 & 18.661 & \multirow{2}{*}{0.478} & \multirow{2}{*}{$0.635^{\mathrm{NS}}$} \\
\hline & & Exptl. & 47.691 & 14.315 & & \\
\hline
\end{tabular}

** - Significant at 1\% level NS - Not Significant

Table 9. Difference in delayed posttest scores in the attributes of sample groups with regard to level of intelligence.

\begin{tabular}{|c|c|c|c|c|c|c|c|c|}
\hline Level of Intelligence & Dependent Varia & les & Group & Size & Mean & SD & t value & $P$ value \\
\hline \multirow{10}{*}{ Low } & \multirow{6}{*}{$\begin{array}{l}\text { Achievement in } \\
\text { Mathematics }\end{array}$} & \multirow{2}{*}{ Knowledge } & $\mathrm{C}$ & 3 & 763190 & 8.248 & \multirow{2}{*}{1.025} & \multirow{2}{*}{$0.352^{\mathrm{NS}}$} \\
\hline & & & $\mathrm{E}$ & 4 & 82.143 & 7.143 & & \\
\hline & & \multirow{2}{*}{ Understanding } & $\mathrm{C}$ & 3 & 63.667 & 11.547 & \multirow{2}{*}{0.274} & \multirow{2}{*}{$0.795^{\mathrm{NS}}$} \\
\hline & & & $\mathrm{E}$ & 4 & 40.000 & 18.257 & & \\
\hline & & \multirow{2}{*}{ Application } & $\mathrm{C}$ & 3 & 47.059 & 11.765 & \multirow{2}{*}{0.624} & \multirow{2}{*}{$0.560^{\mathrm{NS}}$} \\
\hline & & & $\mathrm{E}$ & 4 & 41.176 & 12.707 & & \\
\hline & \multirow{2}{*}{\multicolumn{2}{|c|}{ Interest in Mathematics }} & $\mathrm{C}$ & 3 & 80.688 & 2.291 & \multirow[b]{2}{*}{0.332} & \multirow{2}{*}{$0.754^{\mathrm{NS}}$} \\
\hline & & & $\mathrm{E}$ & 4 & 77.381 & 16.755 & & \\
\hline & \multirow{2}{*}{\multicolumn{2}{|c|}{ Mathematics test Anxiety }} & $\mathrm{C}$ & 3 & 54.040 & 16.895 & \multirow{2}{*}{0.765} & \multirow{2}{*}{$0.479^{\mathrm{NS}}$} \\
\hline & & & $\mathrm{E}$ & 4 & 44.318 & 16.476 & & \\
\hline \multirow{10}{*}{ Average } & \multirow{2}{*}{\multicolumn{2}{|c|}{ Knowledge }} & $\mathrm{C}$ & 15 & 72.381 & 11.411 & \multirow{2}{*}{2.007} & \multirow{2}{*}{$0.055^{\mathrm{NS}}$} \\
\hline & & & $\mathrm{E}$ & 14 & 79.592 & 7.336 & & \\
\hline & \multirow{4}{*}{$\begin{array}{l}\text { Achievement in } \\
\text { Mathematics }\end{array}$} & \multirow{2}{*}{ Understanding } & $\mathrm{C}$ & 15 & 37.333 & 9.611 & \multirow{2}{*}{0.775} & \multirow{2}{*}{$0.445^{\mathrm{NS}}$} \\
\hline & & & $\mathrm{E}$ & 14 & 41.428 & 17.913 & & \\
\hline & & Annlicotion & $\mathrm{C}$ & 15 & 38.431 & 17.346 & \multirow{2}{*}{0.180} & \multirow{2}{*}{$0.858^{\mathrm{NS}}$} \\
\hline & & Appication & $\mathrm{E}$ & 14 & 39.496 & 14.115 & & \\
\hline & \multirow{2}{*}{\multicolumn{2}{|c|}{ Interest in Mathematics }} & $\mathrm{C}$ & 15 & 74.974 & 14.983 & \multirow{2}{*}{0.829} & \multirow{2}{*}{$0.414^{\mathrm{NS}}$} \\
\hline & & & $\mathrm{E}$ & 14 & 70.748 & 12.211 & & \\
\hline & \multirow{2}{*}{\multicolumn{2}{|c|}{ Mathematics test Anxiety }} & $\mathrm{C}$ & 15 & 51.313 & 20.254 & \multirow{2}{*}{0.583} & \\
\hline & & & $\mathrm{E}$ & 14 & 47.511 & 14.059 & & $0.565^{\mathrm{NS}}$ \\
\hline & Knowledge & & $\mathrm{C}$ & 3 & 52.381 & 8.248 & 7000 & 0002 ** \\
\hline High & Knowledge & & $\mathrm{E}$ & 3 & 85.714 & 0.000 & 1.000 & 0.002 \\
\hline Hign & & & $\mathrm{C}$ & 3 & 6.000 & 36.055 & 0555 & $0609^{\mathrm{NS}}$ \\
\hline & Understanding & & $\mathrm{E}$ & 3 & 46.667 & 20.817 & 0.555 & 0.609 \\
\hline
\end{tabular}




\begin{tabular}{|c|c|c|c|c|c|c|c|}
\hline Level of Intelligence & Dependent Variables & Group & Size & Mean & SD & t value & P value \\
\hline & Application & $\begin{array}{l}\mathrm{C} \\
\mathrm{E}\end{array}$ & $\begin{array}{l}3 \\
3\end{array}$ & $\begin{array}{l}54.902 \\
60.784\end{array}$ & $\begin{array}{l}17.971 \\
14.805\end{array}$ & 0.438 & $0.684^{\mathrm{NS}}$ \\
\hline & Interest in Mathematics & $\begin{array}{l}\mathrm{C} \\
\mathrm{E}\end{array}$ & $\begin{array}{l}3 \\
3\end{array}$ & $\begin{array}{l}79.629 \\
86.243\end{array}$ & $\begin{array}{l}18.652 \\
12.329\end{array}$ & 0.512 & $0.635^{\mathrm{NS}}$ \\
\hline & Mathematics test Anxiety & $\begin{array}{l}\mathrm{C} \\
\mathrm{E}\end{array}$ & $\begin{array}{l}3 \\
3\end{array}$ & $\begin{array}{l}40.404 \\
53.030\end{array}$ & $\begin{array}{l}12.247 \\
17.075\end{array}$ & 1.041 & $0.357^{\mathrm{NS}}$ \\
\hline
\end{tabular}

** Significant at 1\% level NS - Not Significant

Table 1 reveals that both the control and experimental groups did not differ significantly in the achievement and interest in mathematics, and mathematics anxiety before the treatment to them.

Table 2 shows that both the control and experimental groups did not differ significantly in the achievement and interest in mathematics, and mathematics anxiety after the treatment to them.

Tables 3-6 indicate that the control and experimental groups scored better in the mathematics post achievement test than in the pre-test. But there was no difference in the mathematics interest scores of control and experimental groups before and after treatment. With regard to the mathematics anxiety, the control group did not show any difference between the pre and post scores, and the experimental group exhibited reduction of anxiety due to the treatment; The students with average level of intelligence showed lesser anxiety in the post-test than in the pre-test.

Table 7 shows that both groups did not show any difference in the gain scores in achievement test, interest and anxiety in mathematics.

Tables 8-9signified that the experimental group students showed better retention capacity to the Knowledge-based questions than that by the control group. This is also true when they have high level of intelligence. No significant difference was found in the delayed post-test in interest and anxiety of both the control and experimental groups. It is also true with regard to the level of intelligence.

\section{Discussion}

In this study, the control group was taught by chalk and talk method and the experimental group was treated with music-blended (western classical violin pieces) mathematics class. The pre-test scores of both the control and experimental groups were equal in their subject knowledge, interest in mathematics, and mathematics anxiety prior to experimentation. It revealed that the two groups were homogeneous.

In post-test also, both the control and experimental groups did not show any significant difference in achievement in mathematics, interest in mathematics, and mathematics anxiety. This might be that both the traditional method and music-blended classroom do not affect the select attributes of students with MLD. The same results prevailed in gain scores of both the control and experimental groups. This result coincides with the studies made by [19] Hoffman (1995), Kluball, (2001), [13] Furnhan and Strbac (2002), Costa
(2004) [10] and [15] Goldenberg et al. (2013) as well. Anyhow, these findings are not aligned with the findings of the many reviewed studies ([36] Whitehead, 2002; Cardarelli, 2004; [26] Merrell, 2004; [29] Olson, 2004; [25] McLelland, 2005; [21] Jones \&Estell, 2007).

In delayed post-test, the control group did not exhibit any difference in the achievement scores in mathematics in terms of learning objectives and level of intelligence whereas the experimental group scored more in the knowledge-based questions. With regard to high level of intelligence also, the experimental group students gained more in the learning objective - knowledge than the control group students. This may be due to the western classical violin music played in a slow tempo set to sound like that it sounds far away from the classroom helped the students to retain more information than the control group. This finding was supported by the study conducted by [6] Campabello et al. (2002). In the delayed post-test, the experimental group did not differ significantly with regard to the learning objectives understanding and application and also for low and average levels of intelligence. This coincides with the reviewed study made by [13] Furnhan \& Strbac (2002).

In mathematics interest scores, both the control and experimental groups did not differ in pre-test, post-test, gain scores and in delayed post-test. This reveals that the instrumental music played in the classroom did not contribute for the mathematics interest of the students with MLD. This may be due to the short span of experimental period, which lasted only for 44 days, about 45 minutes per day.

The experimental group showed lesser post-mathematics test anxiety than the pre--mathematics test anxiety. This may be the result of the violin music-enabled mathematics classroom. This finding coincides with the reviewed studies [17] (Hendricks, 2001; Hirokawa \& Ohira, 2003; [30] Rastogi \& Silver, 2014) also.

The control and experimental groups did not exhibit any difference in the gain in mathematicstest anxiety and delayed anxiety. This indicates that the instrumental music-enabled classroom did not affect the anxiety of the students. This finding is supported by the study conducted by [15] Goldenberg et al. (2012) as well.

\section{Recommendations}

The findings of the study reveal that blending the instrumental music in the mathematics classroom helps the students with MLD to retain the learned information in mathematics and also it helps to overcome the anxiety of the students with MLD towards mathematics. 
Music motivates children to stay focused and study mathematics for longer periods of time, and hence they can be provided with a place to work with music playing in the background in their study room or living room. It is recommended that music be played in the evening time before they start doing their mathematics homework. The home atmosphere exercises a good amount of influence over the emotional stability of such children. Cognition and emotions are closely linked with music and also the human body has a physiological response to the music. So the parents of students with MLD can play music in their home when their child seems to be emotionally disturbed. Parents should not dishearten their children as they lag in their arithmetic ability and they should look for ways to overcome their MLD. As a facilitator, music can stimulate the MLD children to concentrate on arithmetic performance. Music helps focus on accuracy and attention of the students. Learning to play a musical instrument can improve attention, concentration, motivation and memory. It unifies more than one neural pathway by using multi-sensory channels such as visual, auditory and kinesthetic respectively. The parents of children with MLD can make arrangement for music education for progressive development in Mathematics.

It is recommended that, in a classroom setting, when a soft comforting western classical instrumental music (stringed instruments) is played in the background, it might create a perfect harmony in unification of senses. The mathematics teachers can play western classical violin music CDs in the mathematics classroom to unify the senses of students with MLD. This would help the students with MLD to concentrate and perform in their mathematical tasks in the classroom.

Music even outs mental, physical and emotional rhythms to attain a state of deep concentration and focus in which large amounts of content information can be processed and learned. It is advocated that western classical instrumental music that is of 50 to 80 beats per minute creates an atmosphere of focus that leads students into deep concentration in the alpha brain wave state. Alpha brain wave state is a state of physical and mental relaxation, in which fresh creative energy begins to flow and fear vanishes. Also, energizing Western Classical music can assist in holding attention during sleepy times of day and help students stay focused and relaxed in the learning atmosphere. So, it is worthwhile to play western classical music inside the classroom during afternoons and teaching jaded topics.

Since most of the school teachers do not have proper awareness about MLD, they label the students as weak in mathematics or as slow learners. The students with MLD are not slow learners but they are different learners. Labeling them as slow learners would hinder their achievement in mathematics and would affect their self-esteem. So it is responsibility of the teachers to teach them differently as per their needs. It is suggested that teachers of mathematics can come forward to implement music in the classroom of students with MLD so that it can create an environment which would enhance their performance.

\section{Conclusion}

Nowadays, all the schools are being provided with ICT equipment and so it is easy to make the ordinary classroom into music-blended classroom. Indispensable steps to be taken to identify students with MLD at the primary level and they can be provided special instructional strategies in the music-blended classroom. Schools are expected to have musical CDs in excess for this purpose. If every school supports and encourages their wards exposed to MusicMathematics blended teaching, the students in general, especially those with MLD retain the learned information and in turn overcome the anxiety towards mathematics. It is in the hands of the teachers and mentors to implement musicMathematics blended transaction.

\section{Appendix A}

Table 1A. Violin pieces used during the treatment to the experimental group.

\begin{tabular}{|c|c|c|c|}
\hline S. No. & Selected violin pieces & Beats / min & Composer \\
\hline 1. & Concerto for Violin and Orchestra in D, Op. 61, II & Larghetto - 60-66 bpm & Beethoven \\
\hline 2. & The Violin Concerto in D major, Op.35 & Andante- $56-88 \mathrm{bpm}$ & Pyotr Ilyich Tchaikovsky \\
\hline 3. & The Violin Concerto No. 3 in G major & Adagio- 58-97 bpm & Wolfgang Amadeus Mozart \\
\hline 4. & Concerto No. 1 in E, Rv 269 spring & Largo- 42-66 bpm & Antonio Lucio Vivaldi \\
\hline 5. & Violin Concerto No. 4 in D major, K. 218 & Andante - 56-88 bpm & Wolfgang Amadeus Mozart \\
\hline 6. & Concerto No. 5 in A major, K. 218 & Adagio- 58-97 bpm & Wolfgang Amadeus Mozart \\
\hline 7. & Concerto No. 3 in F, Rv 293 autumn & Adagio- 58-97 bpm & Antonio Lucio Vivaldi \\
\hline 8. & Triple Concerto in C Major, Op. 56- II. & Largo- $42-66$ bpm & Beethoven \\
\hline 9. & Violin Concerto in D Op. 61 - II. & Larghetto- $60-66$ bpm & Beethoven \\
\hline 10. & Concerto in D Major for Violin and Orchestra, Op. 77 - I & Adagio- 58-97 bpm & Johannes Brahms \\
\hline 11. & Violin Concerto in D major, Op. 77 & Allegro & Johannes Brahms \\
\hline 12. & Adagio in E major, K. 261 & Adagio- 58-97 bpm & Wolfgang Amadeus Mozart \\
\hline 13. & Concerto No. 5 in A major, K. 218 & Adagio- 58-97 bpm & Wolfgang Amadeus Mozart \\
\hline 14. & Violin Concerto No. 5 In A Major, K. 219 & Adagio- 58-97 bpm & Wolfgang Amadeus Mozart \\
\hline 15. & Violin Concerto in D major, Op. No. 74 & Adagio- 58-97 bpm & Johannes Brahms \\
\hline
\end{tabular}




\section{Appendix B}

Table A1 Difference in Pretest scores in the attributes of sample students with MLD

Table A2 Difference in Posttest scores in the attributes of sample students with MLD

Table A3 Difference between pre-test and post-test scores if the attributes of students in the control group

Table A4 Difference between pre-test and post-test scores in the attributes of students in the control group with regard to level of intelligence

Table A5 Difference between pre-test and post-test scores in the attributes of students in the experimental group

Table A6 Difference between pre-test and post-test scores in the attributes of students in the experimental group with regard to level of intelligence

Table A7 Difference in the gain scores in the attributes of students in the sample groups

Table A8 Difference in the delayed post-test scores in the attributes of students in the sample groups

Table A9 Difference in delayed posttest scores in the attributes of sample groups with regard to level of intelligence

\section{Acknowledgements}

The author acknowledges the support of Ms J. Kirupa Kani, Formerly Teacher Educator in Bethlahem College of Education, Karungal, Kanyakumari District, India in helping collection and analysis of the data for the study.

\section{References}

[1] Adkins, S. (n. d.). Connecting are powers of music to the learning of languages. Retrieved from www.njcu.edu/cill/vol4.

[2] An, S., A., Tillman, D., A., Boren, R., \& Wang, J. (2014). Fostering elementary students' mathematics disposition through music-mathematics integrated lessons. International Journal for Mathematics Teaching \& Learning. Retrieved from

http://connection.ebscohost.com/c/articles/98968289/fostering -elementary-students-mathematics-disposition-through-musicmathematics-integrated-lessons.

[3] Anderson, S., Henke, J., McLaughlin, M., Ripp, M., \& Tuffs, P. (2000). Using background music to enhance memory and improve learning. Retrieved from http://shodhganga.inflibnet.ac.in/bitstream/10603/14702/9/09_ chapter-ii.pdf.

[4] Best, J. W., \& Kahn, J. V. (2012). Research in education $\left(10^{\text {th }}\right.$ ed.). New Delhi: Prentice Hall of India.

[5] Bucholtz, J., Dunham, W., \& MC Guinn, S. (1996). Background music and the behavior of middle-school students with emotional disabilities. Retrieved fromhttp://www.sherimcguinn.com/images/BackgroundMusic MastersProject_Sheri-McGuinn.pdf.

[6] Campabello, N., Carlo, D., Jane, M., O'Neil, J., \& Vacek, M. J. (2002). Musicenhances learning. Retrieved from http://eric.ed.gov/?id=ED471580.

[7] Campbell, D. (1997). The Mozart effect. New York: Avon Books.

[8] Cardarelli, D. M. (2004). The effects of music instrumental training on performance on the reading and mathematics portions of the Florida comprehensive achievement test for third-grade students. DAI, 64(10), 3624-A.
[9] Chamorro-Premuzic, T., Swami, V., Terrado, A., \&Furnham, A. (2009). The effects of background auditory interference and extraversion on creative and cognitive task performance. Retrieved from

http://www.ccsenet.org/journal/index.php/ijps/article/viewFile $14458 / 3770$

[10] Costa, G. E. (2004). Effects of three years of piano instruction on children's academic achievement, school performance and self-esteem. Psychology of Music, 32(2), 139-152.

[11] Doyle, M., \&Furnham, A. (2012). The distracting effects of music on the cognitive test performance of creative and noncreative individuals. Thinking Skills and Creativity, 7(1), 1-7. doi: 10.1016/j.tsc.2011.09.002.

[12] Eaton, J. (2007). The effects of an integrated reading and music instructional approach on fourth-grade students' reading and music achievement. DAI, 67(12), 4492-A.

[13] Furnham, A., \& Strbac, L. (2002). Music is as distracting as noise: The differential distraction of background music and noise on the cognitive test performance of introverts and extraverts. Ergonomics, 42(3), 203-217.

[14] Garrett, H. E. (1961). Statistics in psychology and education. New Delhi: Paragon International.

[15] Goldenberg, M. A., Floyd, A. H. L., \& Moyer, A. (2013). No effect of a brief music intervention on test anxiety and exam scores in college undergraduates. Retrieved from http://www.jasnh.com/pdf/Vol10-No1-article1.pdf.

[16] Haynes, S. E. (2003). The effect of background music on the mathematics test anxiety of college algebra students. DAI, 65(5), 1708-A.

[17] Hendricks, C. B. (2001). A study of the use of music therapy techniques in a group for the treatment of adolescent depression. DAI, 62(2), 472-A.

[18] Hirokawa, E., \&Ohira, H. (2003). The effects of music listening after a stressful task on immune functions, neuroendocrine responses, and emotional states in college students. Journal of Music Therapy, 40(3), 189-211. doi: 10.1093/jmt/40.3.189.

[19] Hoffman, D. S. (1995). Relationship between academic achievement and participation in a comprehensive and sequential keyboard-based public school music education program. DAI, 56(6), 2161-A. 
[20] Jackson, N. (2009). The little book of music for the classroom: Using music to improve memory, motivation, learning and creativity. United Kingdom: Crown House Publishing.

[21] Jones, M. H., \& Estell, D. B. (2007). Exploring the Mozart effect among high school students. Psychology of Aesthetics, Creativity, and the Arts, 1(4), 219-224.

[22] Kang, H. J., \& Williamson, V. J. (2013). Background music can aid second language. Psychology of Music, 42(5), 728747.

[23] Kluball, J. L. (2001). The relationship of instrumental music instruction and academic achievement for the senior class of 2000 at Lee Country high school, Leeburg, Georgia. DAI, 61(11), 4320-A.

[24] Lang, L. L. (2001). The effects of music on the task persistence of multiple disabled children in a self-contained classroom. (Master's Thesis). Rowan University, United States. Retrieved from http://www.researchgate.net/publication/34758577_The effect s_of_music_on_the_task_persistence_of_multiple_disabled_c hildren_in_a_self-contained_classroom_.

[25] McLelland, M. (2005). The impact of instrumental music on student academic achievement. DAI, 65(12), 4503-A.

[26] Merrell, A. (2004). The benefits of incorporating music in the classroom. Retrieved from http://audreymerrell.net/INTASC/INTASC6/the\%20benefits\% $20 \mathrm{of} \% 20$ incorporating\%20music.pdf.

[27] Montello, L., \& Coons, E. E. (1998). Effects of active versus passive group music therapy on preadolescents with emotional, learning, and behavioural disorders. Journal of Music Therapy, 35(1), 49-67.

[28] Nering, M. E. (2002). The effect of piano and music instruction on intelligence of monozygotic twins. Retrieved from

http://www.musicschoolunion.eu/fileadmin/downloads/attach ments/Effects of music.doc.

[29] Olson, E. K. B. (2004). Affirming parallel concepts among reading, mathematics, and music through Kodaly music instruction. DAI, 64(12), 4400-A.

[30] Rastogi, R., \& Silver, E. (2014). Association of music with stress, test anxiety, and test grades among high school students. Retrieved from http://www.jyi.org/issue/associationof-music-with-stress-test-anxiety-and-test-grades-amonghigh-school-students/.

[31] Roden, I., Grube, D., Bongard, S., \&Kreutz, G. (2013). Does music training enhance working memory performance? Findings from a quasi-experimental longitudinal study.

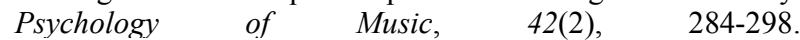
doi: $10.1177 / 0305735612471239$.

[32] Savan, A. (1998). A study of the effect of background music on the behaviour and physiological responses of children with special educational needs. Psychology of Music, 27(2), 138146. doi: $10.1177 / 0305735699272005$.

[33] Schellenberg, E. G. (2004). Music lessons enhance IQ. Psychological Science, 15(8), 511-514.

[34] Stanley, C. J. (1978). Educational and psychological measurement and evaluation. New Delhi: Prentice Hall of India.

[35] Urbantimes (n. d.). What does your musical preference say about your personality?

Retrievedfromhttp://urbantimes.co/2012/12/what-does-yourmusical-preference-say-about-yourpersonality/.

[36] Whitehead, B. J. (2002). The effect of music intensive intervention on mathematic scores of middle and high school students. DAI, 67(8), 2710-A. 